\title{
DATA MANAGEMENT FRAMEWORK OF DRONE-BASED 3D MODEL RECONSTRUCTION OF DISASTER SITE
}

\author{
C. $\operatorname{Kim}^{\mathrm{a}, *}, \mathrm{H}$. Moon ${ }^{\mathrm{a}}$, W. Lee ${ }^{\mathrm{a}}$ \\ ${ }^{\text {a }}$ Korea Institute of Civil Engineering and Building Technology, ICT Convergence and Integration Institute, 10223 Goyang-si, \\ Gyeonggi-do, Republic of Korea - (ckim, hsmoon, wslee)@kict.re.kr
}

Commission VI, WG IV/1

KEY WORDS: 3D Model, 3D Reconstruction, Disaster, Drone, Topography, Unmanned Aerial Vehicle (UAV)

\begin{abstract}
:
To rescue peoples in the disaster site in time, information acquisition of current feature of collapsed buildings and terrain is quite important for disaster site rescue manager. Based on information about disaster site, they can accurately plan the rescue process and remove collapsed buildings or other facilities. However, due to the harsh condition of disaster areas, rapid and accurate acquisition of disaster site information is not an easy task. There are possibilities of further damages in the collapse and there are also difficulties in acquiring information about current disaster situation due to large disaster site and limited rescue resources. To overcome these circumstances of disaster sites, an unmanned aerial vehicle, commonly known as a drone is used to rapidly and effectively acquire current image data of the large disaster areas. Then, the procedure of drone-based 3D model reconstruction visualization function of developed system is presented.
\end{abstract}

\section{INTRODUCTIONS}

\subsection{Research Background}

To rescue peoples in the disaster site in time, information acquisition of current feature of collapsed buildings and terrain is quite important for disaster site rescue manager. Based on information about disaster site, they can accurately plan the rescue process and remove collapsed buildings or other facilities. However, due to the harsh condition of disaster areas, rapid and accurate acquisition of disaster site information is not an easy task. There are possibilities of further damages in the collapse and there are also difficulties in acquiring information about current disaster situation due to large disaster site and limited rescue resources.

The advent of unmanned aerial vehicle, coupled with stateof-the-art technologies such as spatial information technology, materials of UAV and rechargeable batteries, provide engineers with unprecedented opportunities to overcome the current processes of site management. First and second challenge is difficulties in accessing disaster sites and difficulties in acquiring information about disaster sites, respectively. Because of the risks of additional collapse of the building by other equipment or people who walk over the ruins, it is not easy task to access the location where people are buried under the ruins. Third and fourth challenge in the disaster area is the difficulties in rapid acquisition of buried people location and difficulties in in acquiring life and death information about buried people. Due to harsh conditions of disaster areas, understanding of regional and geographic information is critical issue for disaster managers. However, because of difficulties in acquiring the geographical information of the disaster sites such as large disaster sites and limited capability of rescue workers, comprehensive site investigation of current location of survivors buried under the remains of the building is not an easy task for disaster managers. The last one is the needs for new technologies for rescuing buried people.

\subsection{Literature Reviews}

The UAV - also known as drone - with sensors have been widely used to get information of the construction site or various facilities. Siebert and Teizer (2014) used UAV technologies to rapidly acquire $3 \mathrm{D}$ data of earthwork construction site. Skycatch Inc. (2015) developed drones and additional facilities for monitoring solar panels and modules to manage solar farms. Liu et al. (2015) developed BIM (Building Information Model)-based progress monitoring framework using the camera-equipped drone. Zhang and Elaksher (2012) developed a drone-based imaging system for $3 \mathrm{D}$ measurement of unpaved road surface distresses.

\subsection{Research Objectives}

The objective of this study is to develop data management framework of drone-based 3D model reconstruction of disaster site. This paper starts with brief description of system architecture. Second, procedure of drone-based 3D model reconstruction is discussed. Then, visualization function of developed system is presented. Lastly, research contributions and recommendations are summarized.

\section{SYSTEM FRAMEWORK}

\subsection{System Architecture}

The developed system is consisting of four subsystem: drone module, depth map generation module, $3 \mathrm{D}$ viewer module, and volume analysis module (Kim et al. 2015). The drone module is equipped with various sensors and stereo-vision camera. The function of this module is to acquire image data of the disaster

* Corresponding author 
site. The depth map generation module develop depth map of the disaster site. The 3D viewer module visualize the $3 \mathrm{D}$ terrain model and 3D collapsed building model. Lastly, the volume analysis module calculate volume of the 3D terrain model and $3 \mathrm{D}$ collapsed building model.

\subsection{Drone Hardware}

The stereo-vision equipped drone has GPS (Global Positioning Systems) module, AHRS (Attitude and Heading Reference System) and IMU (Inertial Navigation System) module to locate the current position and movement of the vehicle. To install the stereo-vision camera module DJI Ronin Gimbal is used and two lithium-ion cell $(16,000 \mathrm{mAh})$ are installed to provide the power to the drone. For the stereo vision camera module, two Point Grey cameras $(1600 \times 1200,59 F P S)$ are installed. This module also has function of wireless data transfer (Wireless Local Area Network) to receive the saved image data of the disaster site and $\log$ data of the drone.

\subsection{D Model Reconstruction Processes}

The first step of the drone-based 3D model reconstruction processes is to acquire disaster site image data from the stereovision camera equipped drone. In this process, stereo image data and location information of the drone are automatically saved on the server of the drone module. Then, depth map of the disaster site is developed to generate $3 \mathrm{D}$ point cloud model of the disaster site. Next, using triangulated irregular network algorithm, the point cloud data of the disaster site is converted into the $3 \mathrm{D}$ model of the disaster site. The detailed processes of the system are presented in the next section.

\section{DRONE-BASED 3D MODEL RECONSTRUCTION OF DISASTER SITES}

\subsection{D Model Generation}

Prior to generate the depth map of the disaster site, log file from drone and image data from the stereo-vision camera, in other words image data from the left side camera and image data from the right side camera are required. Then the epipolar line matching process is conducted to generate the depth map of the disaster site (Figure 1). In this process, feature point extraction is conducted to adjust image difference between image data from the left side camera and right side camera(Figure 2).

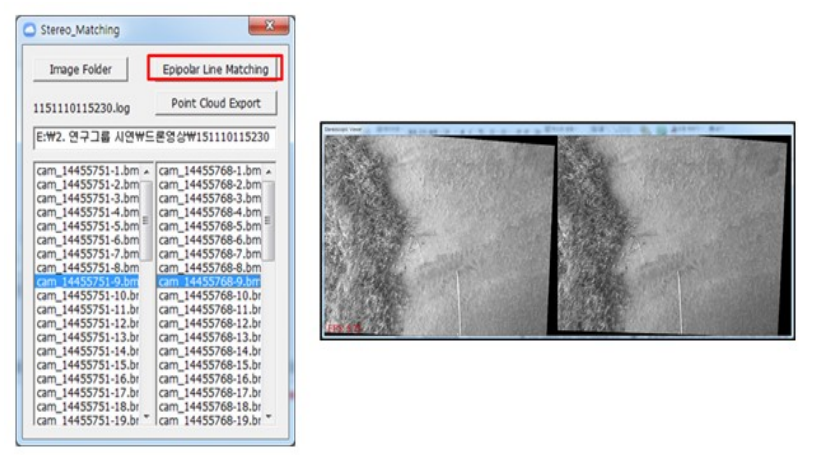

Figure 1. Epipolar Matrix Calculation

After depth map generation from the stereo image matching process, the depth map is converted into the point cloud data
(Figure 3). Lastly the 3D model of the disaster site is generated (Figure 4). To generate the 3D coordination of 3D model of the disaster site, the information about the image data and location data such as focal length, pixel size and base line between two cameras are utilized.

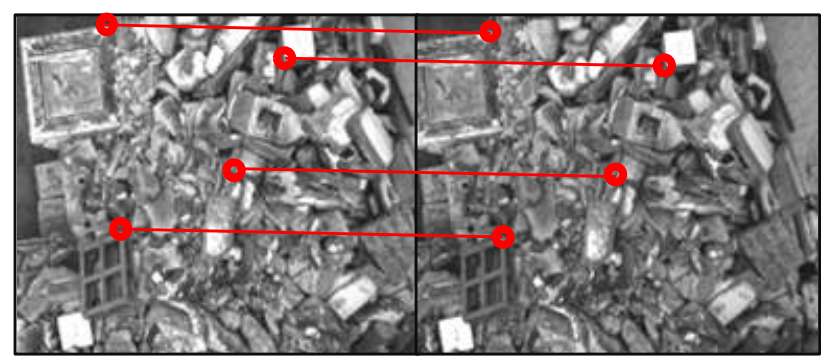

Figure 2. Feature Point Extraction between Image Data from Left Side and Right Side of the Stereo-vision Camera
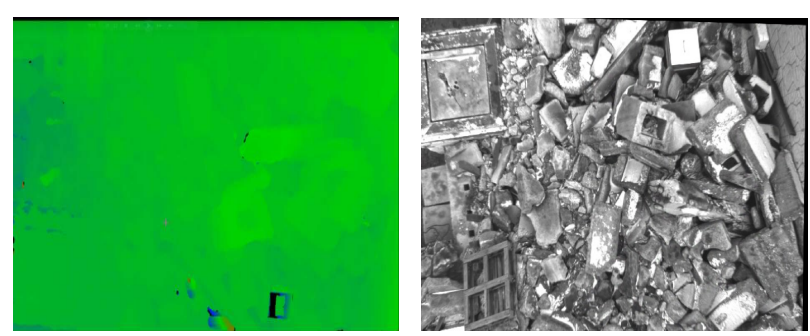

Figure 3. Depth Map (Left) and 3D Point Cloud Model (Right) of the Disaster Site

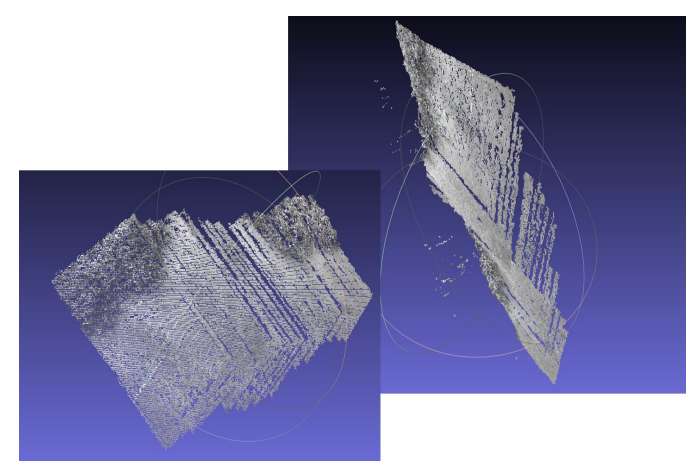

Figure 4. 3D Model of the Disaster Site

\subsection{D Model Viewer}

To effectively visualize the 3D model of the disaster site, the 3D model viewer is developed using Unity engine.

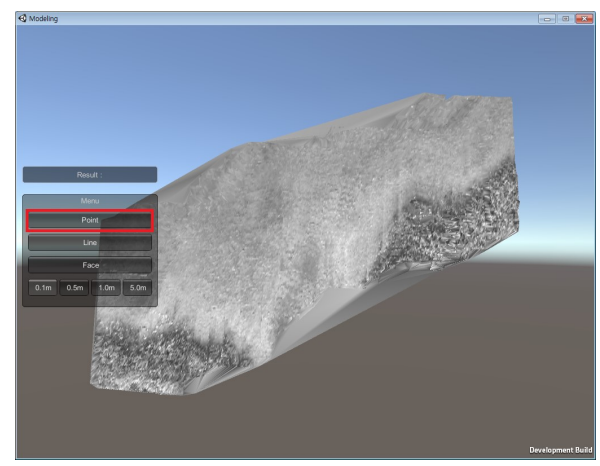

Figure 5. 3D Model Viewer 
The 3D model viewer have function of providing information of 3D point coordination, calculation of distance between two points, and calculation of selected area.

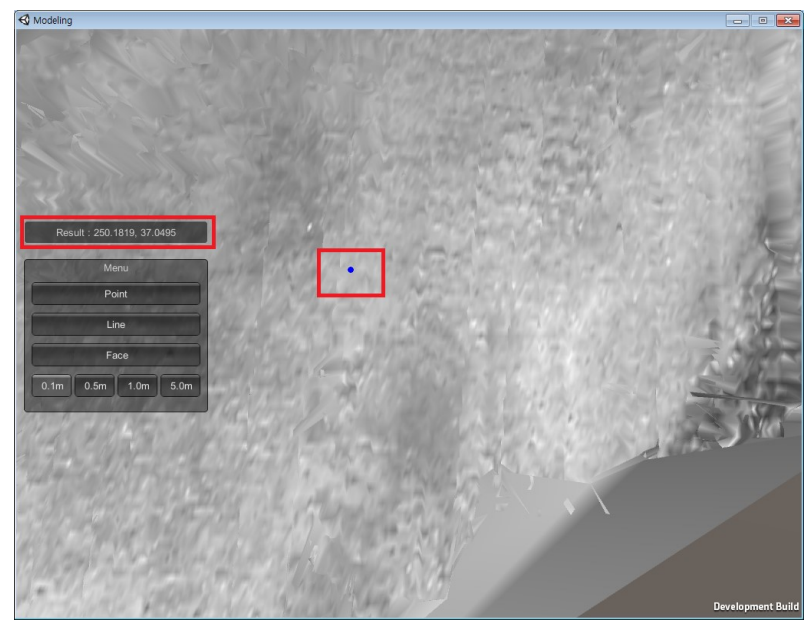

Figure 6. Visualize 3D Point Coordination

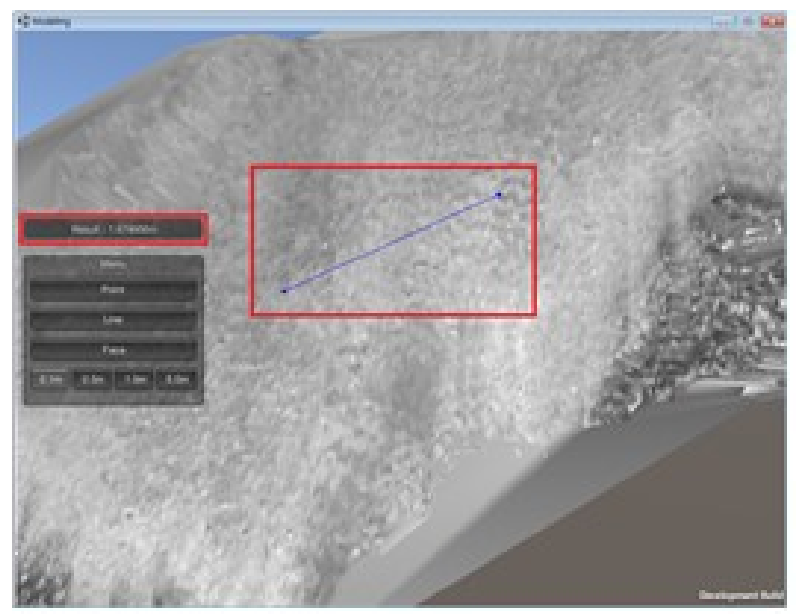

Figure 7. Measurement of Distance between Two Points

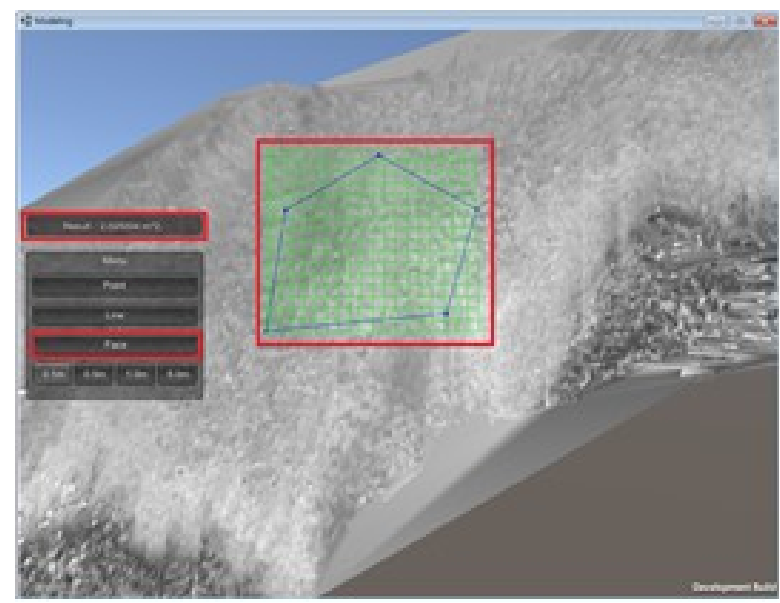

Figure 8. Calculation of Selected Area
In the $3 \mathrm{D}$ point coordination information function, the $\mathrm{X}, \mathrm{Y}, \mathrm{Z}$ coordination of the selected point is presented on the result window, when user click the point button on the menu and then click the point. To measure the distance between two points, click the line button on the menu and then click the two points on the disaster site 3D model. The calculation result of the distance measurement is presented on the screen. Lastly, to calculate the area of the selected region, click the face button on the menu and then the user can printed grid on the 3D model of the disaster site. Next, click the points on the grid and made the closed polygon. The value of the selected region's area is calculated and presented on the screen.

\section{CONCLUSIONS AND RECOMENDATIONS}

This study presented the data management framework of dronebased 3D model reconstruction of disaster site. First, this paper presented the system framework of system architecture and drone system. Then, the processes of the developing depth map and point cloud model of the disaster site are described. Lastly the $3 \mathrm{D}$ model generation and the functions of the $3 \mathrm{D}$ model viewer are presented. For the future study, the field tests should be required to confirm the accuracy and applicability of the developed system.

\section{ACKNOWLEDGEMENTS}

This work was supported by National Research Foundation of Korea grant funded by the Korean Government (NRF2014R1A1A2056217) and "Development of 3D model reconstruction technology of disaster area and location detection technology of survivors buried under the remains of the building" project funded by Korea Institute of Civil Engineering and Building Technology (KICT).

\section{REFERENCES}

Liu, P., Chen, A. Y., Huang, Y.-N., Han, J.-Y., Lai, J.-S., Kang S.-C., Wu, T.-H., Wen, M.-C., and Tsai M.-H., 2014, A Review of Rotorcraft Unmanned Aerial Vehicle (UAV) Developments and Applications in Civil Engineering, Smart Structures and Systems. 13(6), pp. 1065-1094.

Siebert, S. and Teizer, J., 2014, Mobile 3D mapping for surveying earthwork projects using an Unmanned Aerial Vehicle (UAV) system, Automation in Construction, 41, pp. 114.

Skycatch Inc. 2015. Track Your Solar Farms Remotely and Autonomously, retrieved September 1, 2015, from https://www.skycatch.com/solar.html.

Zhang C. and Elaksher A. 2012. An Unmanned Aerial VehicleBased Imaging System for 3D Measurement of Unpaved Road Surface Distresses. Computer-Aided Civil and Infrastructure Engineering, 27(2), pp. 118-129. 